\title{
The genus Raphignathus (Acari: Raphignathidae) from China
}

\author{
QING-HAI FAN ${ }^{1} \&$ XIN-MING YIN ${ }^{2}$ \\ ${ }^{1}$ Department of Plant Protection, Fujian Agricultural University, Fuzhou 350002, China; e-mail: qhfan@pub2.fz.fj.cn \\ ${ }^{2}$ Department of Plant Protection, Henan Agricultural University, Zhenzhou 450001, China
}

\begin{abstract}
The genus Raphignathus from China is reviewed. The morphology of $R$. zhaoi $\mathrm{Hu}$, Jing \& Liang is studied. Four new species of Raphignathus are described: $R$. aciculatus Fan from moss in Fujian (southeastern China), $R$. membranus Fan from fallen leaves in Beijing (north China), R. evidus Fan from moss in Beijing and $R$. hsiufui Fan from moss in Fujian and from tea (Camellia sinensis) in Zhejiang adjecent to Fujian. A key to the known species from China is provided.
\end{abstract}

Key words: Acari, Raphignathus, new species, China

\section{Introduction}

The modern concept of the genus Raphignathus includes species that were formerly assigned to the genera Caligonus Canestrini, Syncaligus Berlese, Acheles Oudemans and Raphignathus Dugès. In the past our understanding of this genus was nebulous due to abbreviated descriptions and the loss of type specimens. Species of Caligonus were moved to other genera and the name of this genus was discarded. Although Berlese (1910) stated that Syncaligus was based on Caligonus petrobius Canestrini, according to his earlier description (Berlese, 1894), this species has the characters of the genus Raphignathus: confluent coxae, fused chelicerae and peritremes situated between gnathosoma and podosoma. For these reasons Oudemans (1923) and Grandjean (1944) treated Syncaligus as a junior synonym of Raphignathus. Oudemans (1903) erected a monotypical genus, Acheles, later distinguishing it from Raphignathus by the separation between coxae II and coxae III (Oudemans 1927). However, in his unpublished figures of the type species, Acheles mirabilis, Oudemans (1903) drew adjacent coxae II and III. It follows that Acheles is a synonym of Raphignathus (Robaux 1976, Meyer \& Ueckermann 1989).

In this paper we review the genus Raphignathus from China and add four new species. Type specimens are deposited in the Department of Plant Protection, Fujian Agricultural University. Measurements are in micrometers.

\section{Genus Raphignathus Dugès}

Raphignathus Dugès, 1834, 22; Oudemans, 1923, 138; Oudemans, 1927, 260; Meyer \& Ryke, 1960, 228; Atyeo, Baker \& Crossley, 1961, 15; Vainstein \& Kuznetsov, 1978, 149; Chaudhri, Akbar \& Rasool, 1979, 193; Zaher \& Gomaa, 1979, 198; Meyer \& Ueckermann, 1989, 28.

Acheles Oudemans, 1903, 101; Oudemans, 1927, 260; Meyer \& Ryke, 1960, 231.

Syncaligus Berlese, 1910, 202.

Type species: Raphignathus ruberrimus Dugès, 1834 
Diagnosis. Body soft to sclerotized, lengths 280-600; red or yellow in life. Chelicerae fused to form a stylophore; peritremes club-like, arising from mid-basal part of stylophore, projecting to anterior margin of podosoma. Palptibial claw small, at most one half length of palptarsus. Subcapitulum with 2 pairs of subcapitular setae and 2 pairs of adoral setae. Dorsum with 3 podosomal shields and an opisthosomal shield; a pair of eyes situated on lateral podosomal shields, no postocular bodies. Dorsum with 11-12 pairs of setae. Ventrally, coxae II adjacent to coxae III. Two pairs of aggenital setae in front of genital pore, which located on a shield and has 3 pairs of setae. Anal pore clearly separated from genital shield, with 3 pairs of setae. Empodium with 2 rows of 4 or more pairs of tenent hairs. Tarsus I with 2 solenidia $\omega$. Number of male and female solendia on tarsi often similar. Dorsal body shields of male somewhat fused.

The mites of this genus are found on tree barks, in litter, moss, soil, in stored products and bird nests and have a world wide distribution.

\section{Key to species of genus Raphignathus Dugès from China}

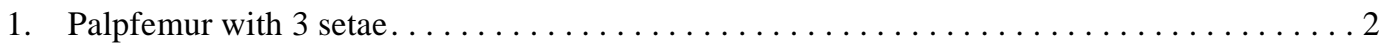

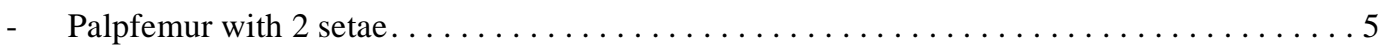

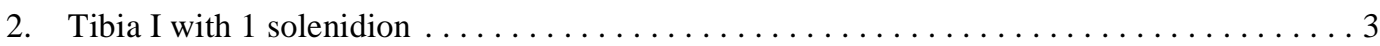

Tibia I with 2 solenidia $\ldots \ldots \ldots \ldots \ldots \ldots$ collegiatus Atyeo, Baker \& Crossley

3. Femur IV with 3 setae $\ldots \ldots \ldots \ldots \ldots \ldots \ldots \ldots \ldots \ldots \ldots \ldots \ldots \ldots \ldots \ldots \ldots$

Femur IV with 2 setae $\ldots \ldots \ldots \ldots \ldots \ldots \ldots$. zhaoi Hu, Jing \& Liang

4. Tarsus II with 16 setae and 1 solenidion; Length of dorsal body setae from 24 to 28 $\ldots \ldots \ldots \ldots \ldots \ldots \ldots \ldots \ldots \ldots \ldots \ldots \ldots \ldots \ldots \ldots \ldots \ldots \ldots \ldots$ aciculatus Fan sp. nov.

- $\quad$ Tarsus II with 15 setae and 1 solenidion; Length of dorsal body setae from 38 to $50 \ldots \ldots \ldots$

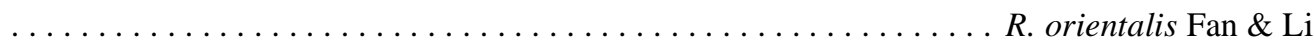

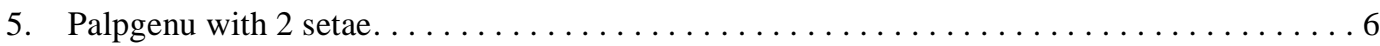

Palpgenu with 1 seta $\ldots \ldots \ldots \ldots \ldots \ldots \ldots \ldots \ldots$ membranus Fan sp. nov.

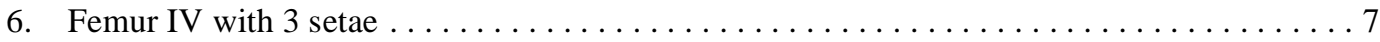

Femur IV with 2 setae $\ldots \ldots \ldots \ldots \ldots \ldots \ldots \ldots \ldots$. hsiufui Fan sp. nov.

7. Lateral podosomal shield small, $c_{2}$ situated on membrane . . . . . . . R. evidus Fan sp. nov.

- Lateral podosomal shield normal, $c_{2}$ situated on this shield . . . . . . . R. gracilis (Rack)

\section{Notes on Raphignathus zhaoi Hu, Jing \& Liang (Figs. 1-24)}

Raphignathus zhaoi Hu, Jing \& Liang, 1995, 23-25.

Raphignathus hongchengensis Hu \& Chen, 1998, 15-18. syn. nov.

The present authors examined this species decribed by Hu et al. (1995) and discovered that there are 3 palpfemoral setae, instead of 2, as originally stated. Hu and Chen (1998) established another new species, Raphignathus hongchengensis, whose distinguishing characters were a striated stylophore (also present in $R$. zhaoi) and the palpfemoral setation. It follows that $R$. hongchengensis is a junior synonym of $R$. zhaoi, a very common species in south China that occurs on tree barks, in moss and in the soil (Fig. 1).

GNATHOSOMA (Figs. 2-4, 7-8; Table 1)

In all stages, stylophore densely covered with veins that arise from basal upper part of subcapitulum. Movable digits needle-like with curved bases and inserted in subtip of chelicerae. Fixed digits reduced to thin sheath. Palpal supracoxal setae, elcp, present in all stages. Counts of setae and so- 
lenidia from trochanter to tarsus of palp in larva: $0,2,1,3+1$ claw, $4+1 \omega+4$ eupathidia; in protonymph: $0,2,2,3+1$ claw, 4+1 $\omega+4$ eupathidia; in deutonymph and in adult: $0,3,2,3+1$ claw, $4+1 \omega+4$ eupathidia. Adoral setae $a d_{1}$ and $a d_{2}$ on subdistal subcapitulum in all stages and often branched. Subcapitular setae $m$ present after protonymph and $n$ present after deutonymph.
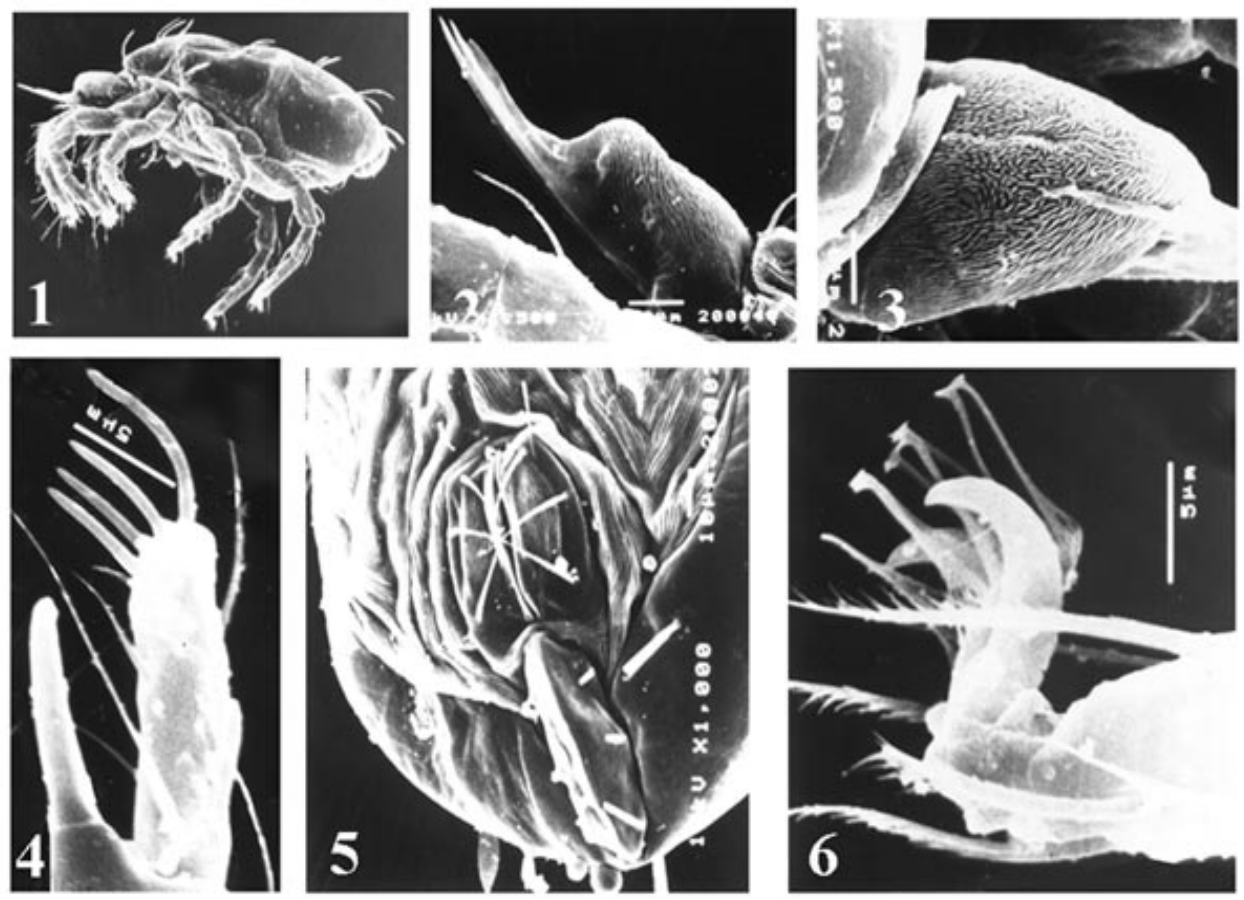

FIGURE 1-6. Raphignathus zhaoi. (female). 1, lateral view of body; 2 , lateral view of stylophore; 3 , dorsal view of stylophore; 4, palp tarsus; 5; genital and anal region; 6, claws and empodium of leg IV.

TABLE 1. Ontogenetic patterns of setae and solenidia on the palp and subcapitulum in Raphignathus zhaoi.

\begin{tabular}{lcccc}
\hline & Larva & Protonymph & Deutonymph & Adult \\
\hline Palp & & & & \\
Femur & $d, l$ & $d, l$ & $d, l, p l$ & $d, l, p l$ \\
Genu & $d$ & $d, l$ & $d, l$ & $d, l$ \\
Tibia & $d, l, v a$ & $d, l, v a$ & $d, l, v a$ & $d, l, v a$ \\
Tarsus & $b p, l p, b a, v a$, & $b p, l p, b a, v a, a c m \zeta$, & $b p, l p, b a, v a, a c m \zeta$, & $b p, l p, b a, v a, a c m \zeta$, \\
Subcapitilar setae & $a c m,(u l \zeta), s u l, \omega$ & $(u l \zeta), s u l \zeta, \omega$ & $(u l \zeta), s u l \zeta, \omega$ & $(u l \zeta), s u l \zeta, \omega$ \\
\hline
\end{tabular}

\section{DORSUM (Figs. 9-13; Table 2)}

Dorsum covered by one median podosomal shield, a pair of lateral podosomal shields and one opisthosomal shield in all stages, except in adult male (all shields confluent). Shields finely punctated. Membrane between podosomal shields and opisthosomal shield variable in stages. Larva with widest membrane. Membrane wider in protonymph than in deutonymph and adult female. In adult male membrane reduced to a small band behind lateral shield. Ontogenetic patterns of dorsal body 
shields, setae and lyrifissures shown in Table 2 . Dorsal setae gradually broadened from larva to adult except $c_{1}, d_{1}, e_{1}, h_{1}$ which in male slender and short.

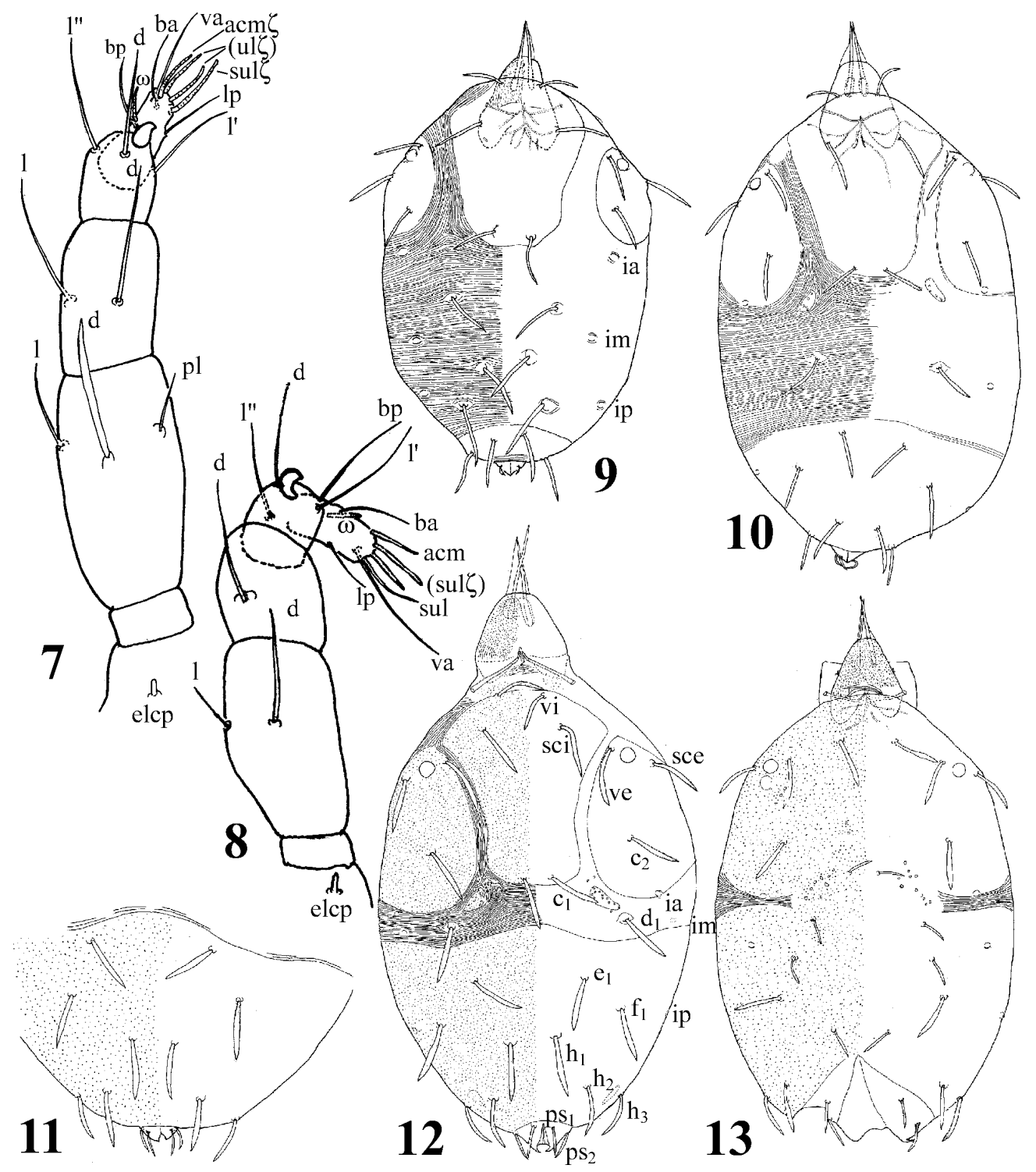

FIGURES 7-13. Raphignathus zhaoi. 7, left palp of female; 8, left palp of larva; 9, dorsum of larva; 10, dorsum of protonymph; 12, dorsum of adult female; 13, dorsum of adult male; 11, dorsal opisthosoma of deutonymph.

\section{VENTER (Figs. 5, 14-20; Table 3)}

Podosomal venter covered by 2 pairs of punctated endopodal shields around inner sides of coxae I-II and coxae III-IV (coxae III in larva), respectively. Coxae II conjunct with coxae III. All shields with finely punctations. Genital pore present only in female deutonymph and female adult. Ontogenetic patterns of ventral, aggenital and genital setae shown in Table 3.

Copulatory male organ consisting of 3 parts, a funnel-like basiphallus, a bulb-like mesophallus and a complex distiphallus which includes an aedeagus, a pair of parameres and a pair of harpagones. 
TABLE 2. Ontogenetic patterns of the setae on the dorsum of Raphignathus zhaoi.

\begin{tabular}{|c|c|c|c|}
\hline & Opisthosoma shield & Number of setae & Lyrifissures \\
\hline Larva & reduced, with $h_{1}, h_{2}$ & 11 pairs & ia, im \& ip on membrance \\
\hline Protonymph & reduced, with $e_{1}, f_{1}, h_{1}, h_{2}$ & 11 pairs & $\begin{array}{l}\text { ia \& ip on shields, im on mem- } \\
\text { brance }\end{array}$ \\
\hline Deutonymph & nomal, with $e_{1}, f_{1}, h_{1}, h_{2}, h_{3}$ & 12 pairs & $\begin{array}{l}\text { ia \& ip on shields, im on mem- } \\
\text { brance }\end{array}$ \\
\hline Adalt (female) & nomal, with $e_{1}, f_{1}, h_{1}, h_{2}, h_{3}$ & 12 pairs & $\begin{array}{l}\text { ia \& ip on shields, im on mem- } \\
\text { brance }\end{array}$ \\
\hline Adalt (male) & $\begin{array}{l}\text { confluent with podosomal } \\
\text { shields, with } e_{1}, f_{1}, h_{1}, h_{2}, h_{3}\end{array}$ & 12 pairs & $i a$, im \& ip on shields \\
\hline
\end{tabular}

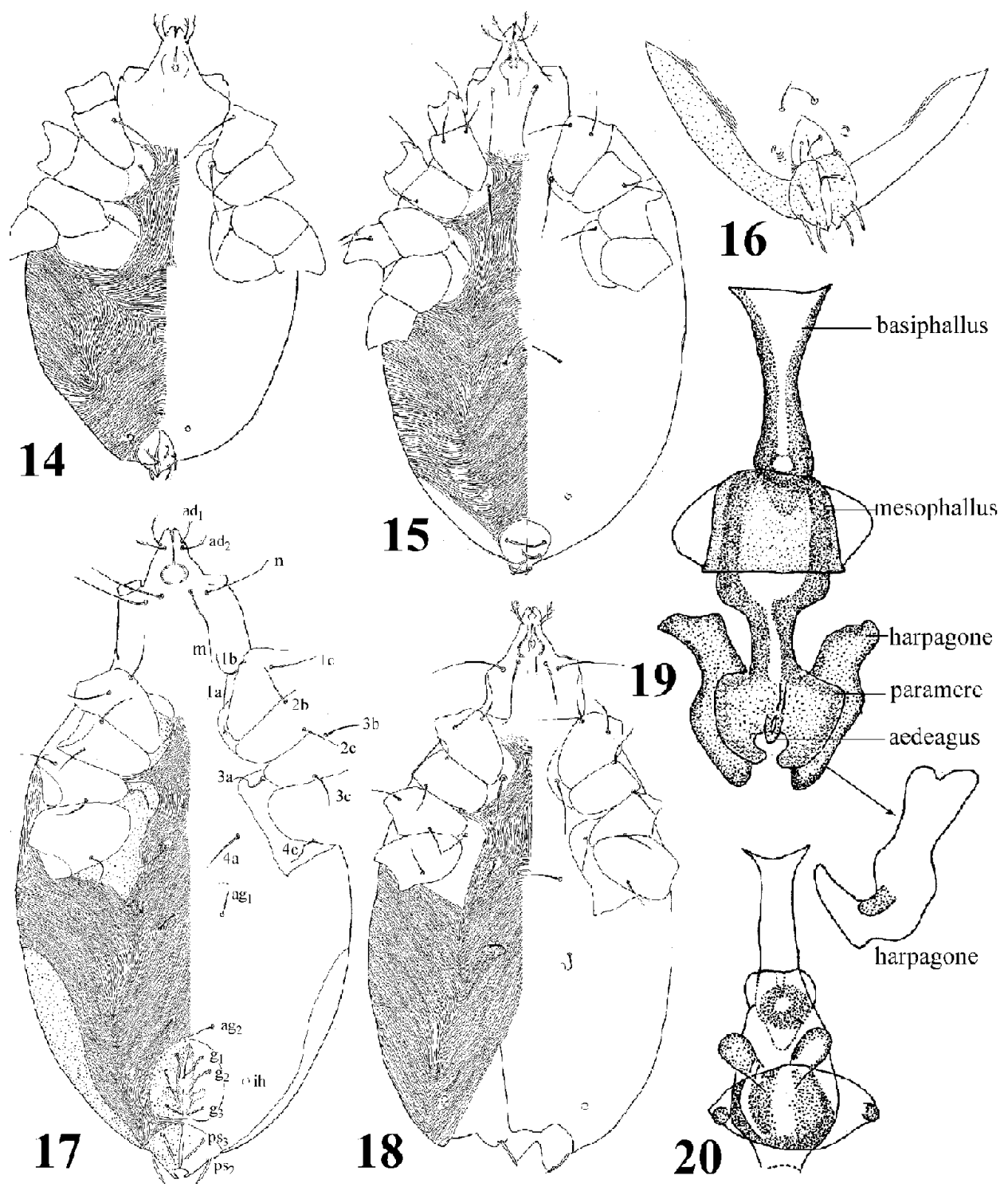

FIGURES 14-20. Raphignathus zhaoi. 14, venter of larva; 15, venter of protonymph; 17, venter of adult female; 18 , venter of male; 16 , ventral opisthosoma of deutonymph; 19 , dorsal view of copulatory organ; 20 , ventral view of front part of copulatory organ. 
TABLE 3. Ontogenetic patterns of the setae on the venter of Raphignathus zhaoi.

\begin{tabular}{lcccccc}
\hline & Larva & Protonymph & $\begin{array}{c}\text { Deutonymph } \\
\text { (female) }\end{array}$ & $\begin{array}{c}\text { Deutonymph } \\
\text { (male) }\end{array}$ & $\begin{array}{c}\text { Adalt } \\
\text { (female) }\end{array}$ & $\begin{array}{c}\text { Adalt } \\
\text { (male) }\end{array}$ \\
\hline Ventral setae & $1 a, 3 a$ & $1 a, 3 a, a g_{1}$ & $1 a, 3 a, 4 a$ & $1 a, 3 a, 4 a$ & $1 a, 3 a, 4 a$ & $1 a, 3 a, 4 a$ \\
Aggenital setae & - & $a g_{1}$ & $a g_{1}, a g_{2}$ & $a g_{1}$ & $a g_{1}, a g_{2}$ & $a g_{1}$ \\
Genital setae & - & - & $g_{1}$ & - & $g_{1}, g_{2}, g_{3}$ & - \\
\hline
\end{tabular}

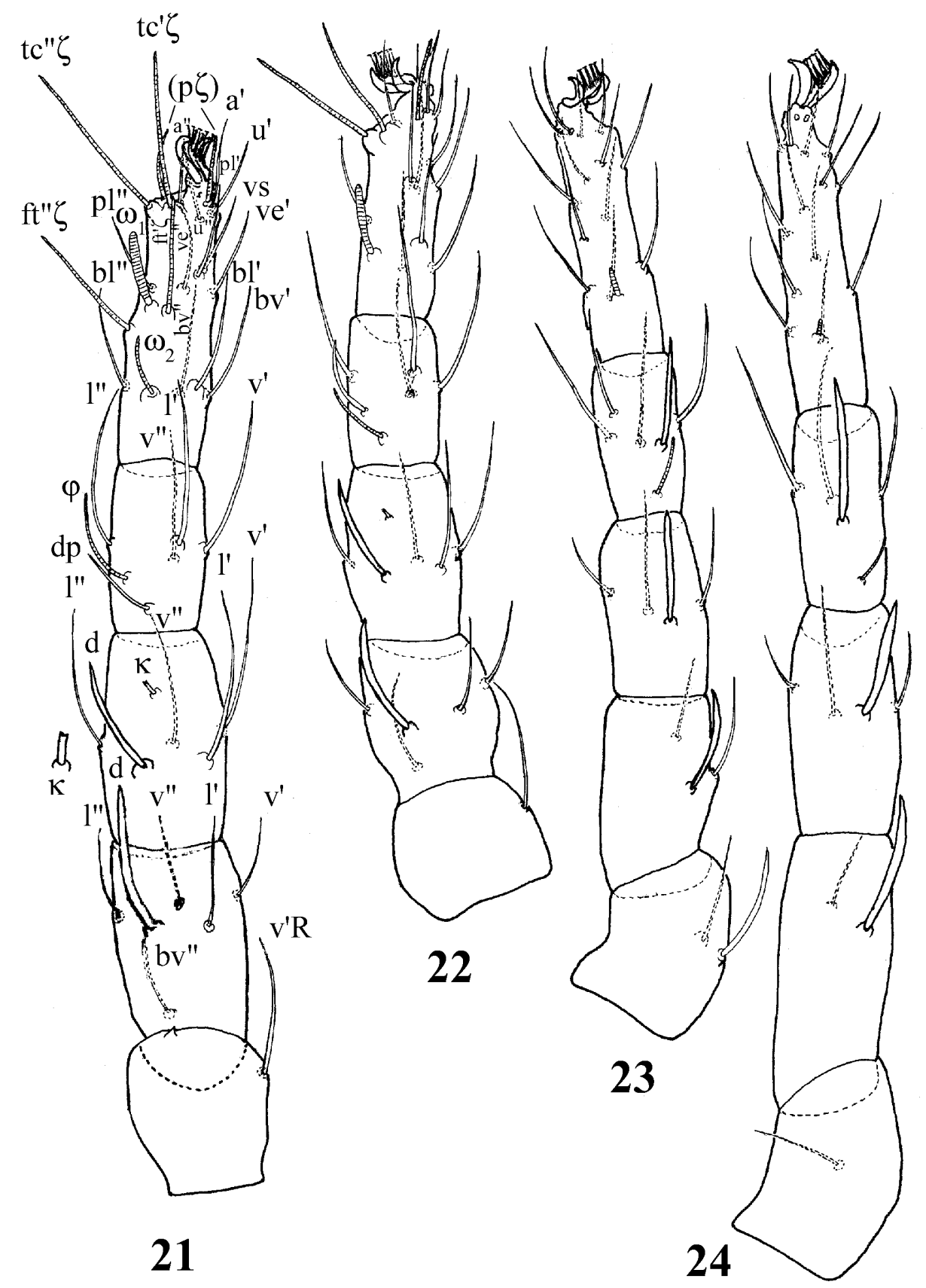

FIGURES 21-24. Raphignathus zhaoi. (female). 21, leg I; 22, leg II; 23, leg III; 24, leg IV. 
TABLE 4. Ontogenetic patterns of setae and solenidia on legs of Raphignathus zhaoi.

\begin{tabular}{|c|c|c|c|c|}
\hline & Larva & Protonymph & Deutonymph & Adult \\
\hline \multicolumn{5}{|l|}{ LEG I } \\
\hline Coxa & $1 b$ & $1 b, 1 c$ & $1 b, 1 c$ & $1 b, 1 c$ \\
\hline Trochanter & - & $v^{\prime} R$ & $v^{\prime} R$ & $v^{\prime} R$ \\
\hline Femur & $d, b v^{\prime \prime}, v^{\prime}$ & $d, b v ”,(v)$ & $d, l, b v^{\prime \prime},\left(v^{\prime}\right)$ & $d,(l), b v^{\prime \prime},(v)$ \\
\hline Genu & $d, l,(v), k$ & $d,(l),(v), k$ & $d,(l),(v), k$ & $d,(l),(v), k$ \\
\hline Tibia & $d p,(l),(v), \varphi$ & $d p,(l),(v), \varphi$ & $d p,(l),(v), \varphi$ & $d p,(l),(v), \varphi$ \\
\hline Tarsus & $\begin{array}{c}p l^{\prime}, v s^{\prime},(v e),(a),(u), \\
(f t),(t c), t c \zeta^{\prime}, p \zeta^{\prime}, \\
\omega_{1}\end{array}$ & $\begin{array}{c}p l^{\prime}, v s^{\prime},(v e),(a),(u), \\
(f t),(t c \zeta),(p \zeta), \\
\omega_{1}, \omega_{2}\end{array}$ & $\begin{array}{l}(b l),(b v),(p l), v s^{\prime} \\
(v e),(a),(u),(f t \zeta) \\
(t c \zeta),(p \zeta), \omega_{1}, \omega_{2}\end{array}$ & $\begin{array}{l}(b l),(b v),(p l), v s^{\prime} \\
(v e),(a),(u),(f t \zeta), \\
(t c \zeta),(p \zeta), \omega_{1}, \omega_{2}\end{array}$ \\
\hline \multicolumn{5}{|l|}{ LEG II } \\
\hline Coxa & - & $2 b$ & $2 b, 2 c$ & $2 b, 2 c$ \\
\hline Trochanter & - & $v^{\prime} R$ & $v^{\prime} R$ & $v^{\prime} R$ \\
\hline Femur & $d, b v^{\prime \prime}, v^{\prime}$ & $d, b v^{\prime \prime}, v^{\prime}$ & $d,(l), b v^{\prime \prime}, v^{\prime}$ & $d,(l), b v^{\prime \prime}, v^{\prime}$ \\
\hline Genu & $d, l,(v), k$ & $d,(l),(v), k$ & $d,(l),(v), k$ & $d,(l),(v), k$ \\
\hline Tibia & $d p,(l),(v), \varphi p$ & $d,(l),(v), \varphi p$ & $d,(l),(v), \varphi p$ & $d,(l),(v), \varphi p$ \\
\hline Tarsus & $\begin{array}{l}p l^{\prime}, v s^{\prime},(v e),(a), \\
(u),(t c), t c^{\prime} \zeta, \omega\end{array}$ & $\begin{array}{l}p l^{\prime}, v s^{\prime},(v e),(a), \\
(u),(t c \zeta), p \zeta, \omega\end{array}$ & $\begin{array}{c}(b l),(b v), p l, v s^{\prime} \\
(v e),(a),(u),(t c \zeta) \\
p \zeta, \omega\end{array}$ & $\begin{array}{c}(b l),(b v), p l, v s^{\prime}, \\
(v e),(a),(u),(t c \zeta) \\
p \zeta, \omega\end{array}$ \\
\hline \multicolumn{5}{|l|}{ LEG III } \\
\hline Coxa & - & - & $3 b, 3 c$ & $3 b, 3 c$ \\
\hline Trochanter & - & $v^{\prime} R, l$ & $v^{\prime} R, l$ & $v^{\prime} R, l$ \\
\hline Femur & $d, v^{\prime}$ & $d, v^{\prime}$ & $d, l, v^{\prime}$ & $d, l, v^{\prime}$ \\
\hline Genu & $d, l, v$ & $d, l^{\prime},(v)$ & $d, l^{\prime},(v)$ & $d, l^{\prime},(v)$ \\
\hline Tibia & $d p,(l),(v), \varphi p$ & $d,(l),(v), \varphi p$ & $d,(l),(v), \varphi p$ & $d,(l),(v), \varphi p$ \\
\hline Tarsus & $\begin{array}{c}v s^{\prime},(v e),(a),(u), \\
(t c), \omega\end{array}$ & $\begin{array}{c}v s^{\prime},(v e),(a),(u), \\
(t c), \omega\end{array}$ & $\begin{array}{c}(b l),(b v), v s^{\prime},(v e) \\
(a),(u),(t c), \omega\end{array}$ & $\begin{array}{c}(b l),(b v), v s^{\prime},(v e), \\
(a),(u),(t c), \omega\end{array}$ \\
\hline \multicolumn{5}{|l|}{ LEG IV } \\
\hline Coxa & - & - & - & $4 b$ \\
\hline Trochanter & - & - & $v^{\prime} R$ & $v^{\prime} R$ \\
\hline Femur & - & - & $d, v^{\prime}$ & $d, v^{\prime}$ \\
\hline Genu & - & $d$ & $d, l^{\prime},(v)$ & $d, l^{\prime},(v)$ \\
\hline Tibia & - & $d,(v), \varphi p$ & $d, l,(v), \varphi p$ & $d, l,(v), \varphi p$ \\
\hline Tarsus & - & $v s^{\prime}, v e^{\prime},(a),(u),(t c)$ & $\begin{array}{c}v s^{\prime},(v e),(a),(u),(t c), \\
\omega\end{array}$ & $\begin{array}{c}(b l),(b v), v s^{\prime},(v e), \\
(a),(u),(t c), \omega\end{array}$ \\
\hline
\end{tabular}

LEGS (Figs. 6, 21-24; Table 4)

Tarsal empodium carrying 4 pairs of tenent hairs directly. Solenidia $\omega$ on male tarsi larger than those of female.

\section{HABITATS}

Barks of Actinidia chinensis Planch, Bischofia javanica B1., Bombax malabaricum DC., Camellia sp., Canarium album (Lour.), Caslanopsis sp., Castanopsis eyeri (Champ. ex Benth), Castanopsis sclerophylla (Lindl.), Casuarina equisetifolia (Forst), Cinnamomum sp., Cunninghamia lanceolata (Lamb.), Dimocarpus longan Lour., Ficus retusa Linn., Grevillea sp., 
Mangifera indica L., Magnolia sp., Pinus massoniana Lamb., Platanus orientalis Linn., Pterocarva stenoptera Dc., Pyrus sp., Sapium sebiferum (L.), Schima superba Gardn. \& Champ, Taxus chinensis var. mairei (Lemee \& Levl), Torreya grandis Fort.; living leaves of Ipomoea cairica (L.); fallen leaves of Castanea mollissima Blume; soil and moss.

\section{DISTRIBUTION}

China (Anhui, Fujian, Guangxi, Henan, Hubei, Jiangsu, Jiangxi, Shanghai, Sichuan and Zhejiang).

\section{Raphignathus gracilis (Rack, 1962)}

Acheles gracilis Rack, 1962, 168: 281.

Raphignathus gracilis (Rack, 1962), Atyeo, 1963, 181-182; Gerson, 1968, 434-435; Vainstein \& Kuznetsov, 1978, 150-151; Zaher \& Gomaa, 1979, 198; Ehara, 1980, 248-249; Kuznetsov \& Petrov, 1984, 99; Meyer \& Ueckermann, 1989, 39-41; Koç \& Ayyildiz, 1996, 210-214.

\section{DIAGNOSIS}

Female. Palptibial claw about one half as long as palptarsus. Counts of setae and solenidia from palpgenu to palptarsi: $0,2,2,3+1$ claw, $4+1 \omega+4$ eupathidia. Membrane between dorsal shields bearing 2 pairs of setae, setae $f_{1}$ close to margin of membrane. Ratios $d_{1}-d_{1}: e_{1}-e_{1}: f_{1}-f_{1}=1.4: 1.6: 1$. Coxae III and IV with endopodal shields. Counts of setae and solenidia on legs I-IV: coxae $1 a+2,2$, $3 a+2,1$, trochanters $1,1,2,1$, femora $6,5,3,3$, genua $5+1 \kappa, 5+1 \kappa, 4,4$, tibiae $5+1 \varphi, 5+1 \varphi p, 5+1 \varphi p$, $4+1 \varphi$ p, tarsi 19+2 $\omega, 15+1 \omega, 13+1 \omega, 13$; tarsi I $\omega_{1} 9$, II $\omega$ 8, III $\omega 6$.

Male. Podosomal shields fused with opisthosomal shield, only marginal areas with striation. All shields with punctations. Ratios $d_{1}-d_{1}: e_{1}-e_{1}: f_{1}-f_{1}=1.5: 1.6: 1$. Tarsus IV with 13 setae and 1 solenidion $\omega ; \mathrm{I} \omega_{1}$, II $\omega$, III $\omega$ and IV $\omega$ about equal in length (12.5).

Specimens examined. 22 females, 9 males, 3 larvae, ex. dried Auricularia auricula-judae; barks of Cinnamomum sp., Cynodon dactylon (L.), Eucalyptrus tereticornis Smith, Platanus orientalis L., Psidium guayava L.; leaves of pteridophyte; soil under Dimocarpus longan Lour.; moss.

Distribution. China (Anhui, Chongqing, Fujian, Hunan, Jiangxii, Shanghai, Sichuan); Egypt, former USSR, Germany, Israel, Japan, South Africa, USA, Turkey.

\section{Raphignathus orientalis Fan \& Li}

Raphignathus orientalis Fan \& Li, 1993, 322-323.

\section{DIAGNOSIS}

Female. Palptibial claw approximately one third as long as palptarsus. Counts of setae and solenidia of palp: $0,3,2,3+1$ claw, $4+1 \omega+4$ eupathidia. Dorsal shields with fine striae. Membrane between shields bearing 2 pairs of setae. Ratios $d_{1}-d_{1}: e_{1}-e_{1}: f_{1}-f_{1}=1: 1.5: 2.1$. Venter with 1 pair of small endopodal shields near coxae III and IV. Counts of setae and solenidia of legs I-IV: coxae $1 a+2,2,3 a+2,1$, trochanters $1,1,2,1$, femora $6,5,3,3$, genua $5+1 \kappa, 5+1 \kappa, 4,4$, tibiae $5+1 \varphi, 5+1 \varphi p$, $5+1 \varphi$ p, $4+1 \varphi$ p, tarsi $19+2 \omega, 15+1 \omega, 13+1 \omega, 13$; tarsi I $\omega_{1} 10$, II $\omega$ 7, III $\omega 5$.

Male. Medial podosomal shield confluent with opisthosomal shield, but separated from lateral podosomal shields. Dorsal body setae $c_{1}, d_{1}, e_{1}, h_{1}$ prominently shorter than others. Ratios $d_{1}-d_{1}$ : $e_{1}-e_{1}: f_{1}-f_{1}=1: 1.4: 1.3$. Genito-anal pore dorso-terminal. Counts of setae and solenidia of legs similar to female except tarsi IV: $13+1 \omega$. Lengths of solenidia on tarsi: I $\omega_{1} 19$, II $\omega 19$, III $\omega 20$, IV $\omega$ 20. 
Specimens examined. 5 females, 1 male, from Auricularia auricula-judae, Pennisetum sp.

Distribution: China (Chongqing, Fujian).

\section{Raphignathus collegiatus Atyeo, Baker \& Crossley}

Raphinathus collegiatus Atyeo, Baker \& Crossley, 1961, 17-20; Kuznetsov \& Petrov, 1984, 100-101; Meyer \& Ueckermann, 1989, 42.

Raphignathus guiyanensis Hu, Jing \& Liang, 1995, 21-23. syn. nov.

\section{DIAGNOSIS}

Female. Counts of setae and solenidia on palp: 0, 3, 2, 3+1 claw, $4+1 \omega+4$ eupathidia. Adoral setae simple, without branches. Body dorsum developed, with fine punctations. Membrane with 1 pair of small shields and 1 pair of setae $\left(d_{1}\right)$. Dorsal body setae 11 pairs. Ratios $d_{1}-d_{1}: e_{1}-e_{1}: f_{1}-f_{1}=$ $1: 1.8: 1.4$. Coxae I, II and III, IV with endopodal shields. Counts of setae and solenidia on legs IIV: coxae $1 a+2,2,3 a+2,1$, trochanters $1,1,2,1$, femora $6,6,4,4$, genua $5+1 \kappa, 5+1 \kappa, 4$, 4, tibiae $5+2 \varphi, 5+1 \varphi p, 5+1 \varphi p, 4+1 \varphi p$, tarsi $19+2 \omega, 15+1 \omega, 13+1 \omega, 13$. Lengths of solenidia on tarsi: $\omega_{1} 7.5$, $\mathrm{II} \omega$ 6, III $\omega 3.5$.

Male. Lateral podosomal shields separate from medial podosomal shield, which confluent with opisthosomal shield. Tarsus IV with 13 setae and 1 solenidion $\omega$.

Specimens examined: 2 females, from moss, bark of Bischofia javanica B1.

Distribution: China (Guizhou, Shanghai), Egypt, former USSR, USA.

Discussion: Atyeo et al. (1961) miscounted the setal numbers as 0, 1, 2, 1 on trochanters and as 6, 5, 4, 4 on femora. Kuznetsov \& Petrov (1984) corrected these faults. Hu et al. (1995) erected a new species, $R$. guiyanensis according to the description of Atyeo et al. (1961). The authors studied the paratype of $R$. guiyanensis and found that it is a junior synonym of $R$. collegiatus.

Raphignathus aciculatus Fan sp. nov. (Figs. 25-32)

FEMALE. Dark red in life. Length of idiosoma 367, width 254

Gnathosoma. Dorsal stylophore with striae, 66 long (including digits), movable digits 54 long. Length of palpus 119 , tibial claw about one half as long as palptarsus. Counts of setae and solenidia (from trochanter to tarsus): 0, 3, 2, 3+1 claw, 4+1 $\omega+4$ eupathidia. Setae on subcapitulum with small prongs, $m 46, n 42$.

Dorsum. Dorsal shields striated and punctated. Medial podosomal shield with 1 pair of lateral ornaments. Each lateral podosomal shield with 1 eye, 3 dorsal setae and 1 lyrifissure ( $i a)$. Membrane with 2 pairs of setae $\left(d_{1}\right.$ and $\left.e_{1}\right), 1$ pair of lyrifissure $(\mathrm{im}$ ) and 1 pair of small shield near podosomal shields. Opisthosomal shield bearing 4 pairs of setae, 1 pair of lyrifissure. Ratios $d_{1}-d_{1}: e_{1}-e_{1}: f_{1}-f_{1}$ $=1$ : 1.6 : 2.2. Lengths of setae: vi 27, ve 28, sci 28 , sce 27, $c_{1} 24, c_{2} 27, d_{1} 24, e_{1} 26, f_{1} 28, h_{1} 28$, $h_{2}$ 27, $h_{3}$ 26; distances between setae: vi-vi 23, vi-ve 66, vi-sci 41, ve-sci 33, sci-sci 59, $c_{1^{-}} c_{1} 26, c_{1^{-}}$ $c_{2}$ 66, $c_{1}-d_{1} 41, d_{1}-d_{1} 53, d_{1}-e_{1} 38, e_{1}-e_{1} 84, e_{1}-f_{1} 51, f_{1}-f_{1} 115, f_{1}-h_{1} 51, h_{1}-h_{1} 33, h_{1}-h_{2} 46, h_{2}-h_{2}$ $66, h_{2}-h_{3} 26$. Anal pore dorso-terminal, with 3 pairs of setae, $p s_{1} 25, p s_{2} 24, p s_{3} 24$.

Venter. Without endopodal shields. Ventral setae $1 a$ (37), $3 a$ (36) close to coxae I and III, respectively, and slightly longer than third pair, $4 a$ 29. Aggenital setae 2 pairs, $a g_{1}$ (29) about 1.7 times of $a g_{2}$ (17). Genital valves punctated, bearing 3 pairs of genital setae; $g_{1}$ (26) slightly longer than $g_{2}$ (23) and $g_{3}$ (23). Lyrifissure (ip) situated on membrane lateral to genital valves.

Legs. Lengths of legs I-IV (from base of trochanter to tip of tarsal claw): 289, 248, 247, 321. Empodium with 4 pairs of tenent hairs. Counts of setae and solenidia on legs I-IV: coxae 1a+2, 2, 
$3 a+2,1$, trochanters $1,1,2,1$, femora $6,5,3,3$, genua $5+1 \kappa, 5+1 \kappa, 4,4$, tibiae $5+1 \varphi, 5+1 \varphi p, 5+1 \varphi p$, $4+1 \varphi$ p, tarsi $19+2 \omega, 16+1 \omega, 13+1 \omega, 13$. Lengths of solenidia on tarsi: I $\omega_{1} 13$, II $\omega$ 10, III $\omega 6$.
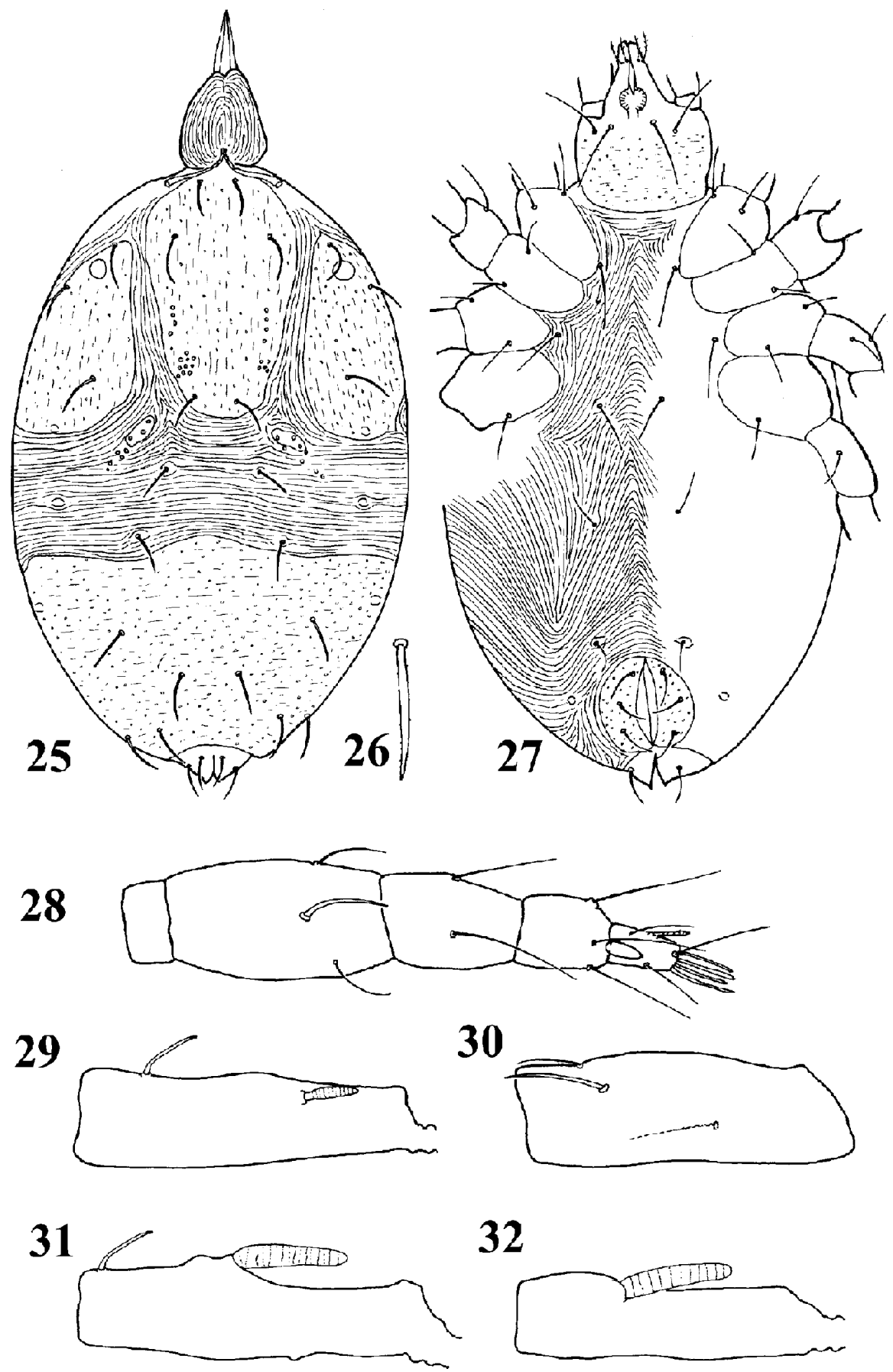

FIGURES 25-32. Raphignathus aciculatus Fan sp. nov. 25, dorsum of female; 26, dorsal seta $f_{1}$ of female; 27 , venter of female; 28, palp of female; 29, solenidia on tarsus I of female; 30, femur IV; 31, solenidia on tarsus I of male; 32, solenidion on tarsus II. 
MALE. Idiosoma 320 long, 207 wide.

Gnathosoma. Stylophore 54 long, movable digit 41 long. Number of setae and solenidia on palp similar to female. Subcapitular setae $m 38$ long, $n 37$.

Dorsum. Dorsal body shields fused, only striated near lateral margin of body. Ratios $d_{1^{-}} d_{1}: e_{1^{-}}$ $e_{1}: f_{1}-f_{1}=1.3: 1.3: 1$. Lengths of setae: vi 23 , ve 23, sci 21, sce 22, $c_{1} 15, c_{2} 21, d_{1} 15, e_{1} 15, f_{1} 20$, $h_{1} 18, h_{2} 25, h_{3} 25$; distances between setae: vi-vi 16, vi-ve 59, vi-sci 30, ve-sce 26, $c_{1}-c_{1} 20, c_{1}$ - $c_{2}$ $59, c_{1}-d_{1} 43, d_{1}-d_{1} 61, d_{1}-e_{1} 31, e_{1}-e_{1} 63, e_{1}-f_{1} 33, f_{1}-f_{1} 48, f_{1}-h_{1} 23, h_{1}-h_{1} 38, h_{1}-h_{2} 30$. Genital pore fused with anal pore, dorso-terminal. 3 pairs of paraproctal setae present, $p s_{1} 27, p s_{2} 14$ and $p s_{3} 20$. Genital setae absent.

Venter. Similar to female, ventral setae 1 a $34,3 a 34,4 a 16$.

Legs. Lengths of legs I-IV: 273, 237, 258, 310. Counts of setae and solenidia similar to female, except tarsi IV: 13+1 $\omega$; I $\omega_{1} 25$, II $\omega$ 25, III $\omega$ 29, IV $\omega 27$.

Etymology. The specific name is from the Latin aciculatus (needle like), referring to the shape of dorsal body setae.

Type materials. Holotype female, allotype male, 4 paratype females, 1 paratype male and 1 paratype deutonymph, from moss, Campus of Fujian Agricultural University, Fuzhou, Fujian, 13 February 1996, Fan.

Remarks. This new species is very close to Raphignathus scutatus Kuznetsov, differing from the latter in: (1) tibiae III with 5 setae and 1 solenidion (with 4 setae and 1 solenidion in the latter); (2) distances between setae $f_{1}-f_{1}>e_{1}-e_{1}>d_{1}-d_{1}$ (in R. scutatus $e_{1}-e_{1}>f_{1}-f_{1}>d_{1}-d_{1}$ ).

Raphignathus membranus Fan sp. nov. (Figs. 33-37)

FEMALE. Idiosoma length 351, width 238 .

Gnathosoma. Stylophore 56 long, movable digits 48 long; palp 102 long, tibial claw approximately one half as long as palptarsus. Counts of setae and solenidia on palp: 0, 2, 1, 3+1 claw, $4+1 \omega+4$ eupathidia. Subcapitulum with 2 pairs of setae, $m$ 32, $n 34$; adoral setae with small prongs.

Dorsum. Dorsal shields developed, with fine punctations. Membrane with 2 pairs of setae. Dorsal body setae stout and short, with fine denticles. Ratios $d_{1}-d_{1}: e_{1}-e_{1}: f_{1}-f_{1}=1.7: 1.3: 1$. Lengths of setae: vi 26, ve 28, sci 25, sce 27, $c_{1} 21, c_{2} 28, d_{1} 21, e_{1} 21, f_{1} 25, h_{1} 26, h_{2} 23, h_{3} 23$; distances between setae: vi-vi 18, vi-sci 46, ve-sci 25, ve-sce 21, $c_{1}-c_{1} 20, c_{1}-c_{2} 84, c_{1}-d_{1} 64, d_{1}-d_{1} 89, d_{1}-e_{1}$ $79, e_{1}-e_{1} 66, e_{1}-f_{1} 53, f_{1}-f_{1} 51, f_{1}-h_{1} 36, h_{1}-h_{1} 21, h_{2}-h_{2} 29, h_{2}-h_{3} 34$.

Venter. With endopodal shields near coxae III and IV. Ventral setae 1a 32, 3a 29, 4a 19. Aggenital setae 2 pairs $a g_{1} 21, a g_{2} 16$. Genital valves with 3 pairs of stout setae, about equal in length, 18. Paraproctal setae stout, $p s_{3} 14, p s_{2} 15, p s_{1} 15$.

Legs. Lengths I-IV: 218, 171, 175, 229. Each tarsal empodium with 5 pairs of tenent hairs. Solenidia on tarsi prominent. Count of setae and solenidia on legs I-IV: coxae $1 a+2,2,3 a+2,1$, trochanters $1,1,2,1$, femora $6,5,3,3$, genua $5+1 \kappa, 5+1 \kappa, 4$, 4, tibiae $5+1 \varphi, 5+1 \varphi p, 5+1 \varphi p, 4+1 \varphi p$, tarsi $19+2 \omega, 15+1 \omega, 13+1 \omega, 13$; tarsi I $\omega_{1} 13$, II $\omega$ 11, III $\omega$ 4.5.

MALE. Unknown.

Etymology. The specific name is from the Latin membran (skin), referring to the striation of the dorsum.

Type material. Holotype female, fallen leaves, Yuanming Park, Beijing, 25 June 1981, Wang.

Remarks. The new species is similar to Raphignathus karrooi Meyer \& Ueckermann, 1989, but differs from in (1) 3 setae on femur IV (the latter with 2) and (2) with 1 solenidion on tarsus III (absent in R. karrooi). 

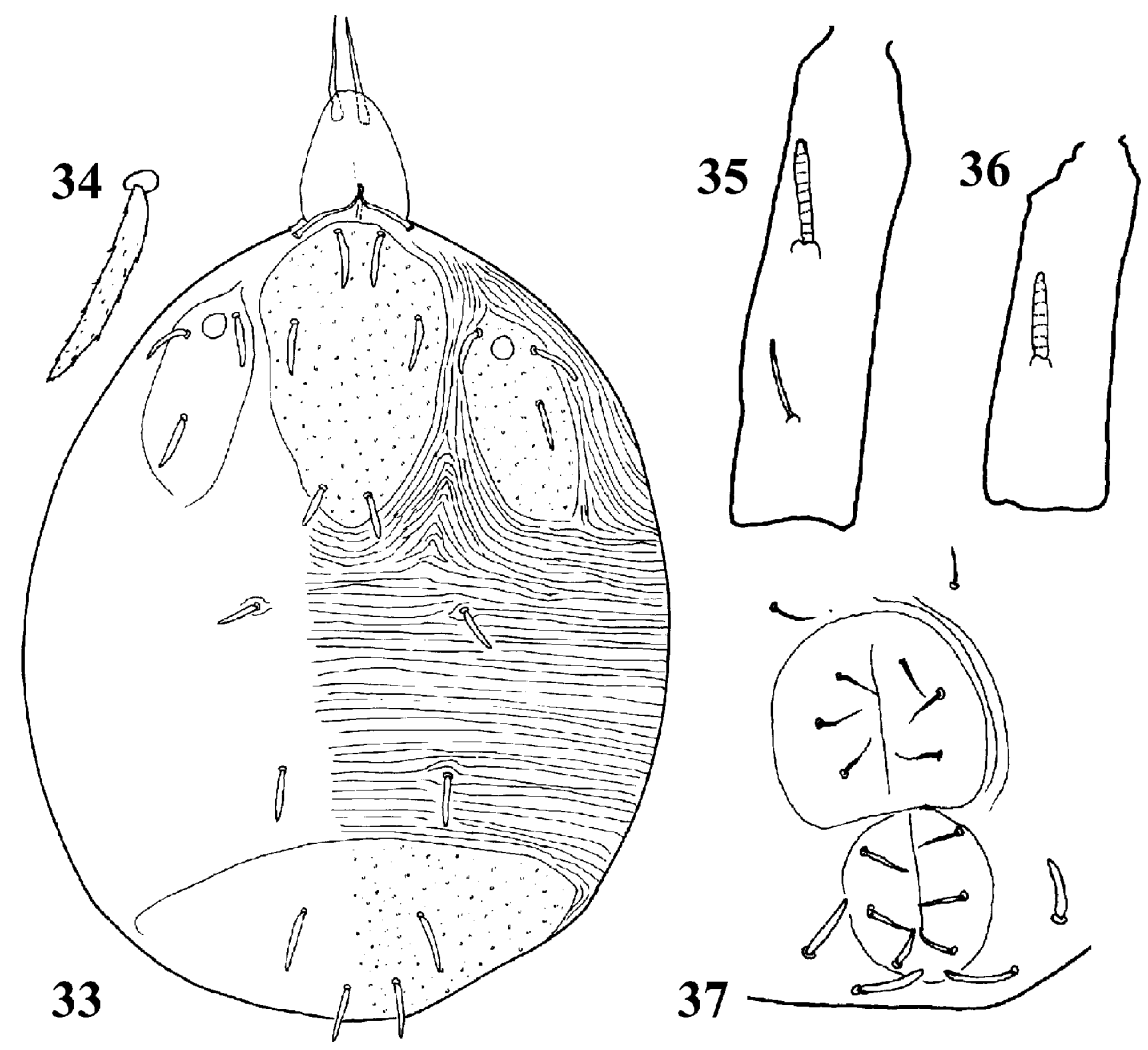

FIGURES 33-37. Raphignathus membranus Fan sp. nov. (female). 33, dorsum; 34, dorsal seta $f_{1}$; 35, solenidia on tarsus I; 36, solenidia on tarsus II; 37, genito-anal region.

\section{Raphignathus evidus Fan sp. nov. (Figs. 38-42)}

FEMALE. Length of idiosoma 331, width 207; red in life.

Gnathosoma. Stylophore 54 long, with fine striae dorsally, movable digit 48 long. Palptibial claw approximately one half as long as palptarsus. Counts of setae and solenidia: 0, 2, 2, 3+1 claw, 4+1 $\omega+4$ eupathidia. Subcapitular setae, $m$ 34, $n$ 35. Adoral setae with small prongs.

Dorsum. Lateral podosomal shields and opisthosomal shield reduced, each bearing only 2 setae, $v e$ and sce. Setae $c_{2}$ situates on membrane. Membrane among shields with 4 pairs of setae, 3 pairs of lyrifissures and 2 pairs of looped bubbles. Seta $h_{1}$ situated on anterior margin of opisthosoma shield. Ratios $d_{1}-d_{1}: e_{1}-e_{1}: f_{1}-f_{1}=1.3: 1.6: 1$. Lengths of setae: vi 25, ve 28, sci 23, sce 29, $c_{1} 23$, $c_{2} 28, d_{1} 25, e_{1} 28, f_{1} 29, h_{1} 28, h_{2} 30, h_{3} 28$; distances between setae: vi-vi 23, vi-sci 30, ve-sci 30 , ve-sce 30, $c_{1}-c_{1} 23, c_{1}-c_{2} 46, c_{1}-d_{1} 51, d_{1}-d_{1} 61, d_{1}-e_{1} 53, e_{1}-e_{1} 77, e_{1}-f_{1} 38, f_{1}-f_{1} 48, f_{1}-h_{1} 25, h_{1}-h_{1}$ $20, h_{1}-h_{2} 26, h_{2}-h_{2} 43, h_{2}-h_{3} 14$.

Venter. Striated and decorated with fine punctations. Endopodal shields present around coxae III and IV. Length of ventral setae: $1 a 37,3 a 33,4 a$ 28. Aggenital setae: $a g_{1} 26, a g_{2} 23$. Genital valves with small punctations, bearing 3 pairs of genital setae, $g_{1} 22, g_{2} 21, g_{3} 20$. Anal pore terminal, lengths of paraproctal setae, $p s_{1} 21, p s_{2} 19, p s_{3} 18$.

Legs. Lengths of legs I-IV: 268, 222, 248, 299. Each tarsal empodium with 4 pairs of tenent hairs. Counts of setae and solenidia on legs I-IV: coxae $1 a+2,2,3 a+2,1$, trochanters $1,1,2,1$, 
femora $6,5,3,3$, genua $5+1 \kappa, 5+1 \kappa, 4,4$, tibiae $5+1 \varphi, 5+1 \varphi p, 5+1 \varphi p, 4+1 \varphi p$, tarsi $19+2 \omega, 15+1 \omega$, $13+1 \omega, 13 ; \operatorname{tarsi} \mathrm{I} \omega_{1} 7$, II $\omega$ 7, III $\omega 3$.

MALE. Unknown.

Etymology. The specific name is from the Latin evidens (distinct), referring to the pattern of dorsal body shields.

Type material. Holotype female, bark of unknown tree, Yuanming Park, Beijing, 9 October 1995, Fan.

Remarks. This species is different from all known species of the genus in: (1) a small lateral podosomal shield, with only 2 setae (ve and sce), $c_{2}$ situated on membrane; (2) a very small opisthosomal shield.
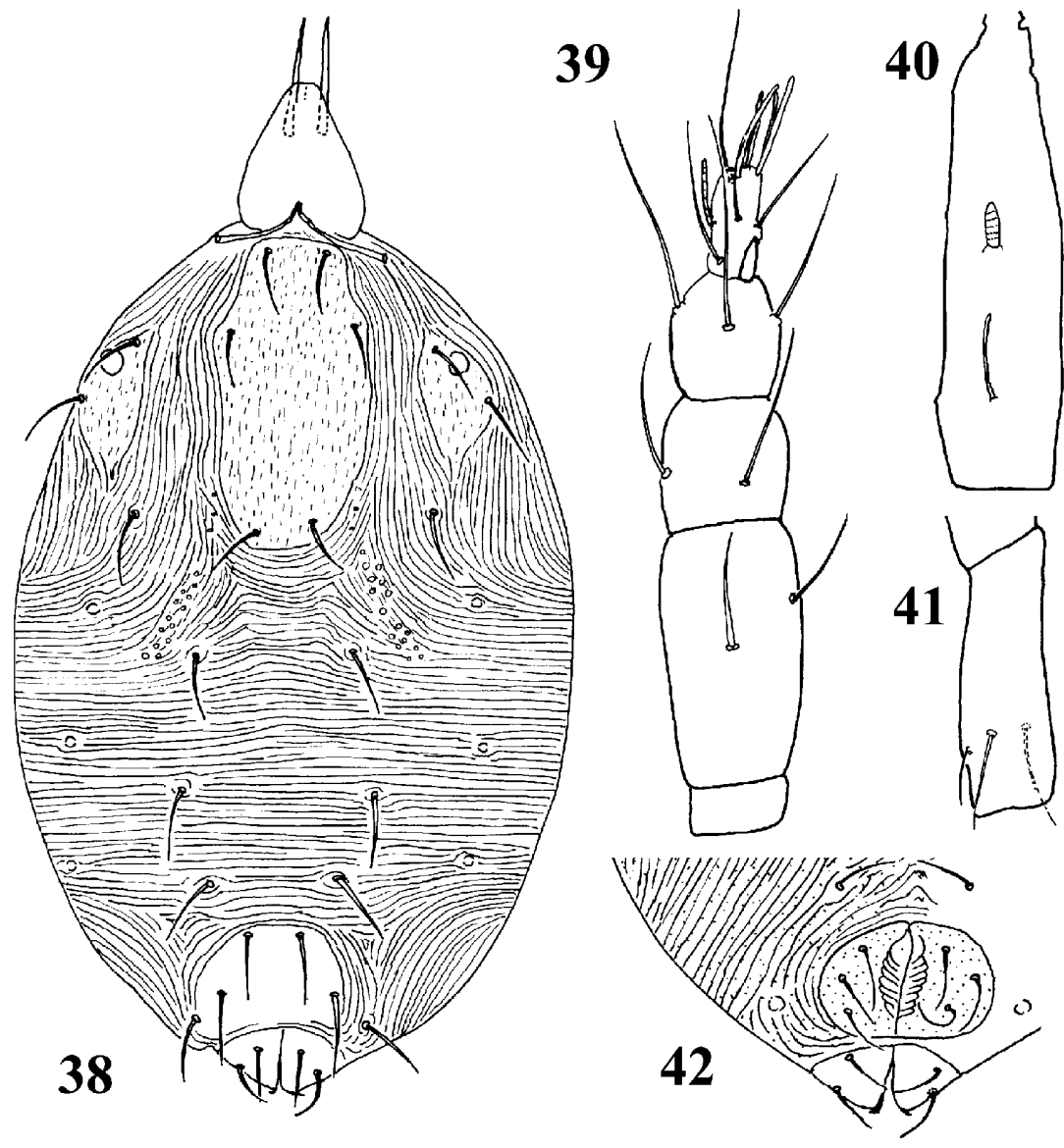

FIGURES 38-42. Raphignathus evidus Fan sp. nov. (female). 38, dorsum; 39, palp; 40, solenidion on tarsus I; 41, femur IV; 42, genital and anal region.

\section{Raphignathus hsiufui Fan sp. nov. (Figs. 43-46)}

Female. Length of idiosoma 331, width 202; dark-red in life.

Gnathosoma. Stylophore 49 long, dorsal surface striated; movable digit 46 long. Palp 109 long, tibial claw small, no more than one third as long as tarsus. Counts of setae and solenidia: 0, 2, 2, $3+1$ claw, 4+1 $\omega+4$ eupathidia. Subcapitular setae 2 pairs, $m 31$, $n 35$; adoral setae with small prongs. 

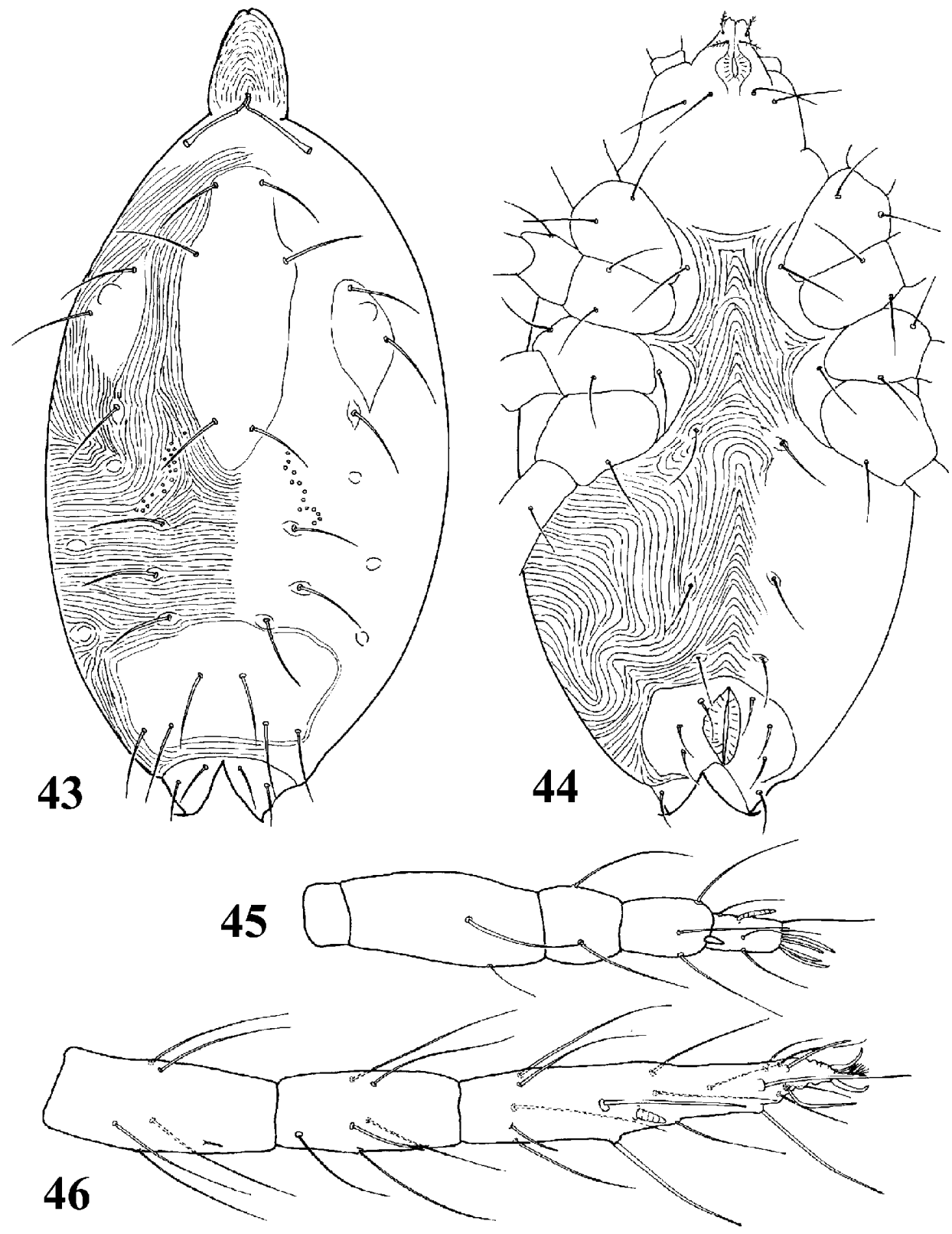

FIGURES 43-46. Raphignathus hsiufui Fan sp. nov. (female). 43, dorsum; 44, venter; 45, palp; 46, genu, tibia and tarsus of leg I.

Dorsum. Lateral podosomal shields reduced, with only 2 setae, setae $c_{2}$ situated on membrane. Membranes bearing 4 pairs of setae $\left(c_{2}, d_{1}, e_{1}, f_{1}\right)$ and 3 pairs of lyrifissures. Setae $f_{1}$ placed on edges of membrane. Opisthosomal shield also reduced, with 3 pairs of dorsal setae, $h_{1}, h_{2}, h_{3}$. Ratios $d_{1^{-}}$ $d_{1}: e_{1}-e_{1}: f_{1}-f_{1}=1.4: 1.5: 1$. Lengths of setae: vi 39, ve 38, sci 38 , sce 39, $c_{1} 35, c_{2} 37, d_{1} 36, e_{1}$ 37, $f_{1} 36, h_{1} 37, h_{2} 39, h_{3} 35$; distances between setae: vi-vi 17, vi-sci 38, ve-sci 21 , ve-sce $30, c_{1}-c_{1}$ 
$20, c_{1}-c_{2} 46, c_{1}-d_{1} 52, d_{1^{-}} d_{1} 65, d_{1^{-}} e_{1} 25, e_{1}-e_{1} 70, e_{1}-f_{1} 17, f_{1}-f_{1} 46, f_{1}-h_{1} 30, h_{1}-h_{1} 21, h_{1}-h_{2} 29 ; h_{2^{-}}$ $h_{2} 41, h_{2}-h_{3} 15$.

Venter. With 2 pairs of endopodal shields near coxae I, II and coxae III, IV. Lengths of ventral setae: $1 a$ 40, $3 a 37,4 a 38$. Aggenital setae 2 pairs, $a g_{1} 35, a g_{2} 29$. Genital shields prominent, bearing 3 pairs of genital setae: $g_{1} 21, g_{2} 18, g_{3} 19$.

Legs. Length of legs I-IV: 294, 258, 289, 310. Counts of setae and solenidia on legs I-IV: coxae $1 a+2,2,3 a+2,1$, trochanters $1,1,2,1$, femora $6,5,3,2$, genua $5+1 \kappa, 5+1 \kappa, 4,4$, tibiae $5+1 \varphi, 5+1 \varphi p$, $5+1 \varphi p, 4+1 \varphi p$, tarsi $19+2 \omega, 15+1 \omega, 13+1 \omega, 3$; tarsi I $\omega_{1} 7$, II $\omega$ 6.5, III $\omega 3$.

MALE. Unknown.

Etymology. The species is named in honour of Prof. Hsiu-fu Chao, Biological Control Institute, Fujian Agricultural University, China.

Type materials. Holotype female, 1 paratype female, from moss, Huaian, Fuzhou, Fujian, 12 October 1994, Fan; 2 paratype females, tea, Lin.

Remarks. The new species is similar to Raphignathus evides sp. nov. in having small lateral and opisthosomal shields, but can be distinguished from the latter by (1) bearing 2 setae on femur IV (the latter with 3) and (2) having longer dorsal body setae (35 to39), mostly extending to bases of setae behind them (much shorter in R. evides, 23 to 31 long, not extending to bases of setae behind them).

\section{Acknowledgements}

The research project was supported by the National Foundation of Natural Science of China.

We are in debt to Prof. Z.-Q. Zhang, Landcare Research, New Zealand for a critical review of the manuscript. We thank Prof. L.-R. Liang and C.-Y. Hu (Fudan University, Shanghai, China) for providing type specimens. We also thank Mr. J.-Z. Lin (Fujian Academy of Agricultural Sciences, Fuzhou, China) for his help in sample collection.

\section{References}

Atyeo, W.T. (1963) New and redescribed species of Raphignathidae (Acarina) and a discussion of the chaetotaxy of the Raphignathoidea. Journal of the Kansas Entomological Society, 36(3), 172-186.

Atyeo, W.T., Baker E.W. \& Crossley D.A. (1961) The genus Raphignathus Dugès (Acarina, Raphignathidae) in the United States with notes on the old World species. Acarologia, 3(1), 14-20.

Berlese, A. (1894) Acari, Myriapoda et Scorpiones ucusque in Italia reperta. Padova, 72, 1.

Berlese, A. (1910) Acari Nuovi-Manipulus V. Redia, 6, 199-214.

Chaudhri, W.M., Akbar, S. \& Rasool, A. (1979) Studies on the predatory leaf inhabiting mites of Pakistan. US Department of Agriculture and Pakistan Agricultural Research Council. PL 480 programme. Project no. PKARS, 30, pp. 139-229.

Dugès, A. (1834) Sur les Acariens. Annales des science naturelle 29 sér. zoologie, 2, 42-45.

Ehara, S. (1980) Illustrations of the mites and ticks of Japan. Zenkoku Noson Kyoiku Kyokai, pp. 248-249.

Fan, Q.-H. \& Li, L.-S. (1993) Descriptions of three new species of superfamily Raphignathoidea (Acari: Actinedida). Journal of Fujian Agricultural University, 22(3), 321-324. (in Chinese with English abstract)

Gerson, U. (1968). Some raphignathoid mites from Israel. Journal of Natural History, 2, 429-437.

Grandjean, F. (1944) Observations sur les acariens de la famille des Stigmaeidae. Archives des Sciences Physiques et Naturelles, 26, 103-131.

Hu, C.-Y., Jing, Z.-Q. \& Liang, L.-R. (1995) Two new species and one new record of the genus Raphignathus Dugès (Acari: Raphignathidae). Journal of Suzhou Railway Teachers College, 12(3), 21-26. (in Chinese with English abstract).

Hu, S. \& Chen, X. (1998) A new species of Raphignathus from Jiangxi, China (Acari: Raphignathidae). Acta Arachnologica Sinica, 7(1), 15-18. 
Koç, K. \& Ayyildiz, N. (1996) Türkiye faunasi için yeni iki Raphignathus Dugès (Acari, Prostigmata, Raphignathidae) Türü. Türkiye Journal of Zoology, 20, 209-214.

Kuznetsov, N.N. \& Petrov, V.M. (1984) Predacious mites of the Baltic region (Parasitiformes: Phytosiidae, Acariformes: Prostigmata). Riga, Zinatne, 90-111.

Meyer, M.K.P. \& Ryke, P.A.J. (1960) Mites of the superfamily Raphignathoidea (Acarina: Prostigmata) associated with South African plants. Annals and Magazine of Natural History, 13(2), 209-234.

Meyer, M.K.P. (Smith) \& Ueckermann, E.A. (1989) African Raphignathoidea (Acari: Prostigmata). Entomology Memoir. Department of Agriculture and Water Supply, Republic of South Africa, 74, 1-58.

Oudemans, A.C. (1903) Acorologische aanteekeningen, VIII. Entomologische Berichten, 14, 100-103.

Oudemans, A.C. (1923) Acarologische aanteekeningen LXX . Entomologische Berichten, 6(129), 138-144.

Oudemans, A.C. (1927) Acarologische aanteekeningen LXXXVIII. Entomologische Berichten, 7(158), $257-$ 263.

Rack, G. (1962) Milben aus Taubennestern mit Beschreibung einer neren Art, Acheles gracilis (Acarina, Raphignathidae). Zoologischer Anzeiger, 168(7-10), 276-292.

Robaux, P. (1976) Observations sur quelques Actinedida (=Prostigmates) du sol d'Amérique du nord. VII. - Sur deux espèces nouvelles de Raphignathidae (Acari). Revue d'Ecologie et de Biologie du Sol, 13(3), 505516.

Vainstein, B.A. \& Kuznetsov, N.N. (1978) Identification key of soil inhabiting mites, Trombidiformes. in Gilyarov M. S. ed. Nauka, Moscow, pp. 147-171.

Zaher, M.A. \& Gomaa, E.A. (1979) Three new species of the genus Raphignathus in Egypt (Prostigmata: Raphignathidae). Acarologia, 21(2), 187-203.

Accepted: 7 May 2000 